\title{
Estimation of remaining water in steel pipes using active thermography
}

\author{
by Y. Kawai*, M. Ishikawa*, H. Nishino*, H. Ishigaki**, K. Ogawa** \\ * Tokushima University, 2-1 Minamijousanjima-cho, Tokushima, 770-8506, Japan, m.ishikawa@tokushima-u.ac.jp \\ ** Toshiba Plant Systems \& Services Corporation, Tsurumi Toushiba-bilding, 4-36-5 Tsutumi-cho, Tsurumi-ku, \\ Yokohama, Kanagawa, 230-8691, Japan
}

\begin{abstract}
The aim of this study is to develop a non-destructive inspection method to estimate the quantity of water remaining in steel pipes by using active thermography. The experimental results showed that variations in the surface temperature of the pipes were dependent on the quantity of the remaining water, which could therefore be estimated by observing the surface temperature. In this study, we propose an effective test setup and a data processing procedure to determine the quantity of remaining water, and present some estimation examples.
\end{abstract}

\section{Introduction}

Nuclear power plants use many pipes for various purposes such as for water supply to a reactor or steam transportation from the reactor to the turbines. These pipes are cut while maintaining or decommissioning the power plants. However, as the water flowing in the pipes may contain radioactive materials, it is important to ensure that there is no water remaining in the pipe before it is cut. The existing hammering or ultrasonic testing methods used for evaluating the remaining water $[1,2,3,4]$ require the presence of an inspector close to the tested pipe; this poses a risk of radiation exposure during the testing process. Therefore, a new and safe method to estimate the remaining water in pipes is desired. The infrared thermographic test is a promising alternative method because it involves non-contact testing and can inspect objects from a distance.

The purpose of this study is to examine the effectiveness of active thermography to estimate the quantity of the remaining water in the pipes. Subsequently, an effective test setup and a data processing procedure to determine the remaining water quantity is proposed.

\section{Numerical investigation of relationship between the remaining water and surface temperature}

In the active thermography test, test objects are heated by a heater, and the surface temperature is observed by an infrared camera. The heat applied to the pipe surface propagates through the thickness and circumferential direction in the pipe, and through the remaining water in the pipe, if any. As the amount of heat conducted into the remaining water depends on its quantity, the temperature of the pipe surface should decrease with increasing quantity of remaining water; hence, the relationship between the surface temperature of the pipe and the remaining water quantity was investigated using numerical calculation.

\subsection{Numerical model}

Numerical calculations were performed using a commercial finite element method solver (ANSYS 18.0). Figure 1 shows a schematic of the numerical model. A heat flux was applied to a stainless steel pipe from the angle of $30^{\circ}$ to the vertical. Table 1 shows the material properties used in the model. The outer diameter of the pipe was $114.3 \mathrm{~mm}$, and the thickness (inner diameter) was varied as shown in Table 2. The heat flux was applied for $60 \mathrm{~s}$ and the post-heating observation time was $240 \mathrm{~s}$ (i.e., the total calculated time was $300 \mathrm{~s}$ ). In the experiments described in the following section, a black body tape of $60 \mathrm{~mm}$ width was stuck on the surface of the pipes to increase the heat input and accuracy of the temperature measurement. Thus, the black body tape was also modelled in the calculations. A heat flux applied to the pipe surface with and without black body tape were $3500 \mathrm{~W} / \mathrm{m}^{2}$ and $1400 \mathrm{~W} / \mathrm{m}^{2}$, respectively (this difference of the heat flux was determined based on that the emissivity of the pipe surface was approximately 0.4 ). The surface heat transfer coefficient $(h)$ was $10 \mathrm{~W} / \mathrm{m}^{2} \mathrm{~K}$. In the calculations, the remaining water was simulated by defining higher heat transfer coefficient in the inner surface of the pipe (see Fig. 1). The $h$ in the water area was calculate from the following equation:

$$
h=\frac{0.15 k}{l} \sqrt[3]{\frac{g \beta P r \Delta T l^{3}}{v^{2}}}
$$

where, $g, \beta, v, k, l$ and $\operatorname{Pr}$ are the gravitational acceleration, the coefficient of volume expansion, the kinematic viscosity, thermal conductivity of water, representative length and the Prandtl number, respectively, and $\Delta T$ denotes the temperature difference between the water and inner pipe surface. The water height $(L)$ from the inner surface was varied as 0 (no water), 
10, 20, and $30 \mathrm{~mm}$. From the calculations, the relationship between the temperature in the black body tape area at the angle of $30^{\circ}$ and $L$ was examined.

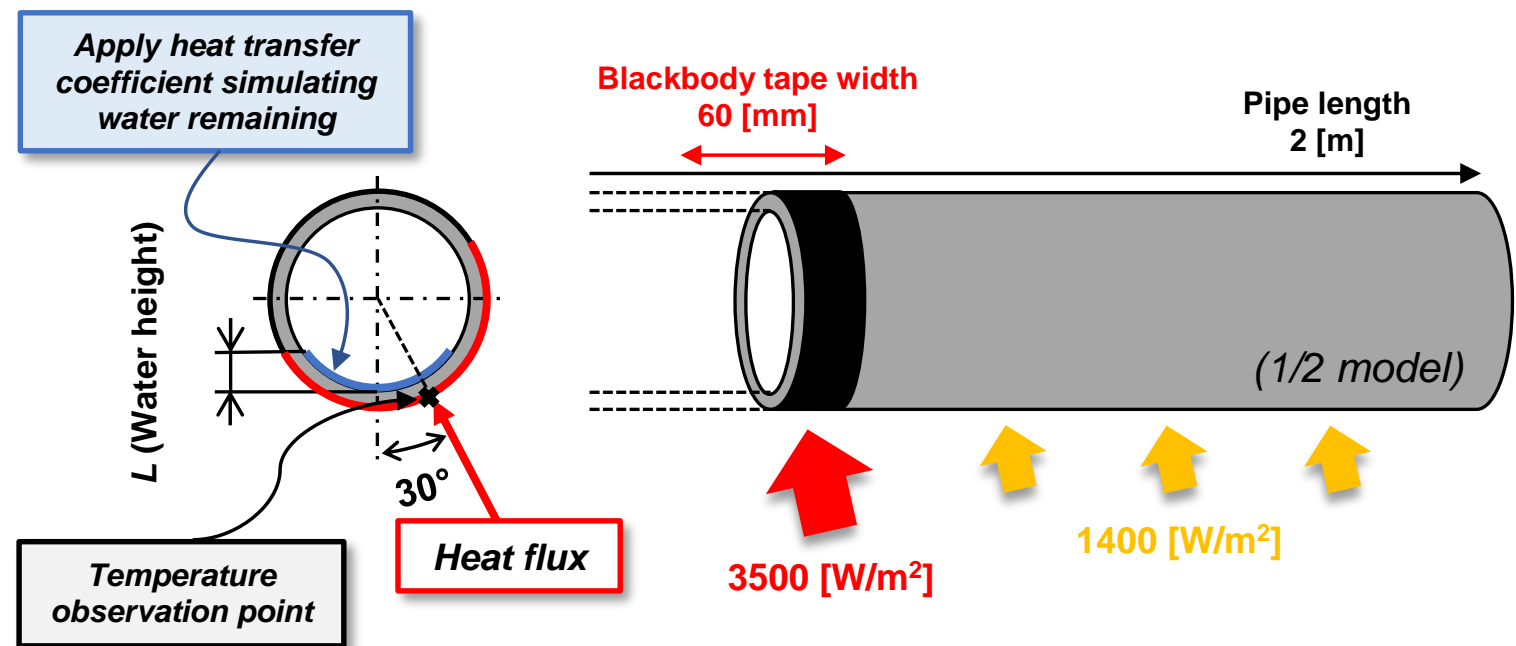

Fig. 1. Schematic of numerical model.

Table 1. Material properties used in calculations.

\begin{tabular}{|c|c|c|c|}
\hline & $\begin{array}{c}\text { Density } \\
{\left[\mathbf{k g} / \mathbf{m}^{3}\right]}\end{array}$ & $\begin{array}{c}\text { Specific heat } \\
{[\mathbf{J} /(\mathbf{k g K})]}\end{array}$ & $\begin{array}{c}\text { Thermal conductivity } \\
{[\mathbf{W} /(\mathbf{m K})]}\end{array}$ \\
\hline Pipe & 7930 & 500 & 16.3 \\
\hline Blackbody tape & 2200 & 1050 & 0.25 \\
\hline
\end{tabular}

Table 2. Outer and inner diameters and pipe thicknesses of the four different calculation models.

\begin{tabular}{|c|c|c|c|}
\hline & $\begin{array}{c}\text { Outer diameter } \\
{[\mathrm{mm}]}\end{array}$ & $\begin{array}{c}\text { Inner diameter } \\
{[\mathrm{mm}]}\end{array}$ & $\begin{array}{c}\text { Pipe thickness } \\
{[\mathrm{mm}]}\end{array}$ \\
\hline 100A sch40 & 114.3 & 102.3 & 6.0 \\
\hline 100A sch80 & 114.3 & 97.1 & 8.6 \\
\hline 100A sch120 & 114.3 & 92.1 & 11.1 \\
\hline 100A sch160 & 114.3 & 87.3 & 13.5 \\
\hline
\end{tabular}

\subsection{Results}

Figure 2 shows the calculated surface temperatures as a function of time for models with four different thicknesses. The results clearly show that the surface temperature dependents on both the pipe thickness and $L$. Therefore, these results indicate that the quantity of the remaining water could effectively be estimated by observing the surface temperature. 

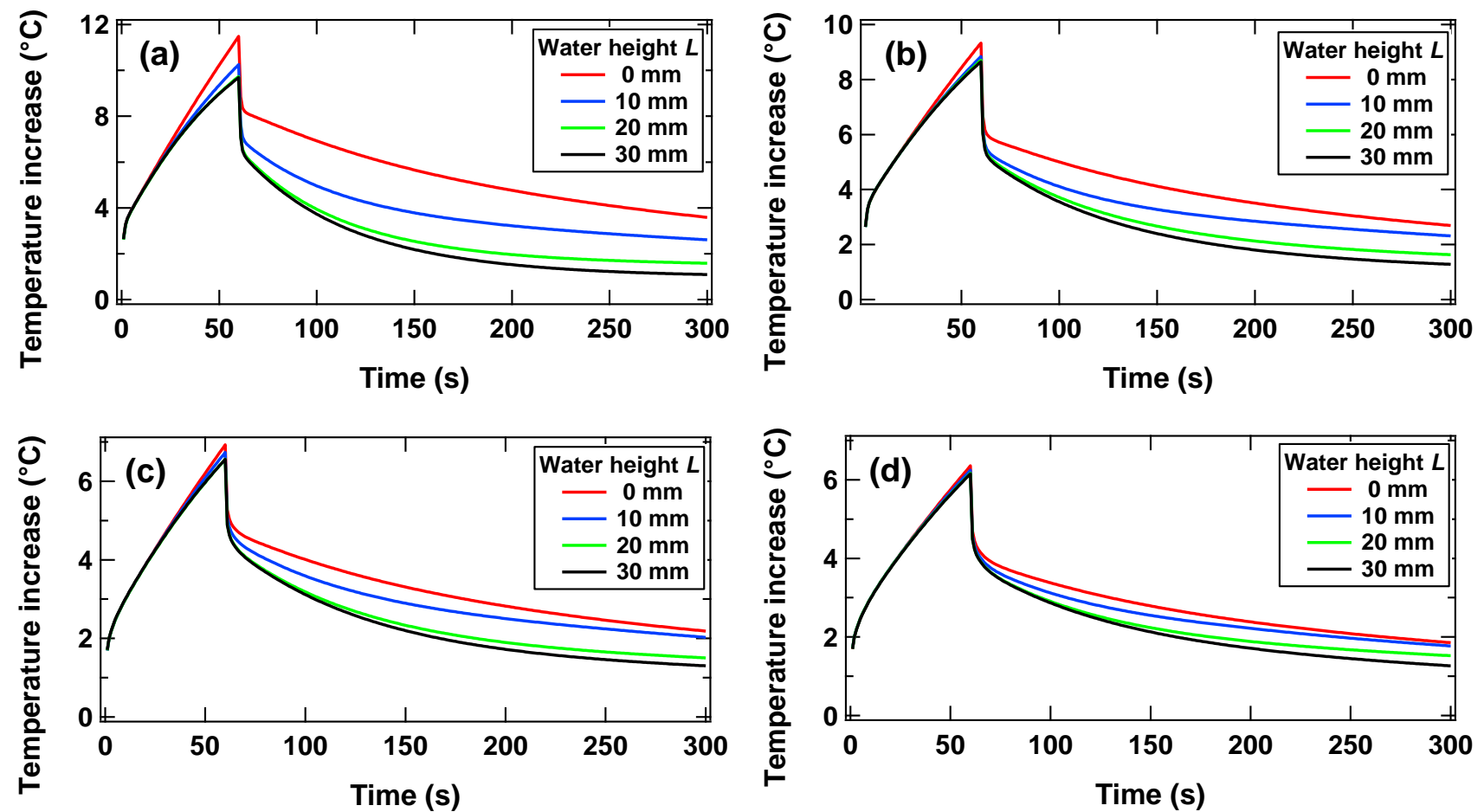

Fig. 2. Numerically calculated surface temperature increase as a function of time for each $L$ for (a) 100A-sch40, (b) sch80, (c) sch120, and (d) sch160.

\section{Experimental verification}

In order to verify the obtained numerical results, experiments for the pipes that have the same thickness as the numerical models were performed.

\subsection{Experimental setup}

Figure 3 shows a schematic of the experimental setup. The test pipes were heated by a halogen line heater (output power: $4 \mathrm{~kW}$ ) from an angle of $30^{\circ}$ with respect to the vertical, and the surface temperature during and after heating was observed from the same angle using an infrared camera (A315, FLIR Systems, Inc.). The heating period was $60 \mathrm{~s}$ and the post-heating observation time was $240 \mathrm{~s}$ (i.e., the total observation time was $300 \mathrm{~s}$ ). A black body tape of $60 \mathrm{~mm}$ width was stuck on the surface of the pipes to increase the heat input and accuracy of the temperature measurement. In the experiments, the height of the remaining water $L$ (see Fig. 3) was varied as 0 (no water), 10, 20, and $30 \mathrm{~mm}$.

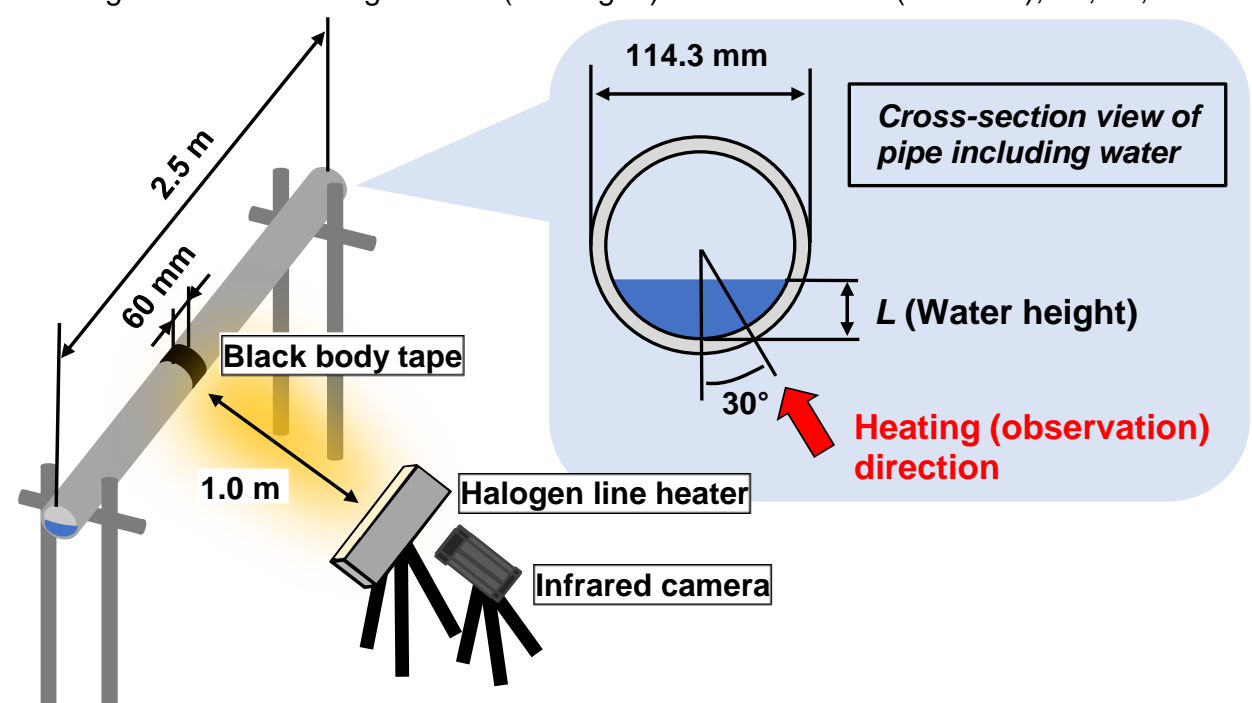

Fig. 3. Schematic of experimental setup. 


\subsection{Experimental results}

Figure 4 shows the observed surface temperature as a function of time for the pipes with four different thicknesses. As compared with the numerical results (figure 2), the experimental results agree well with the numerical results. These results show the validity of the numerical calculations.
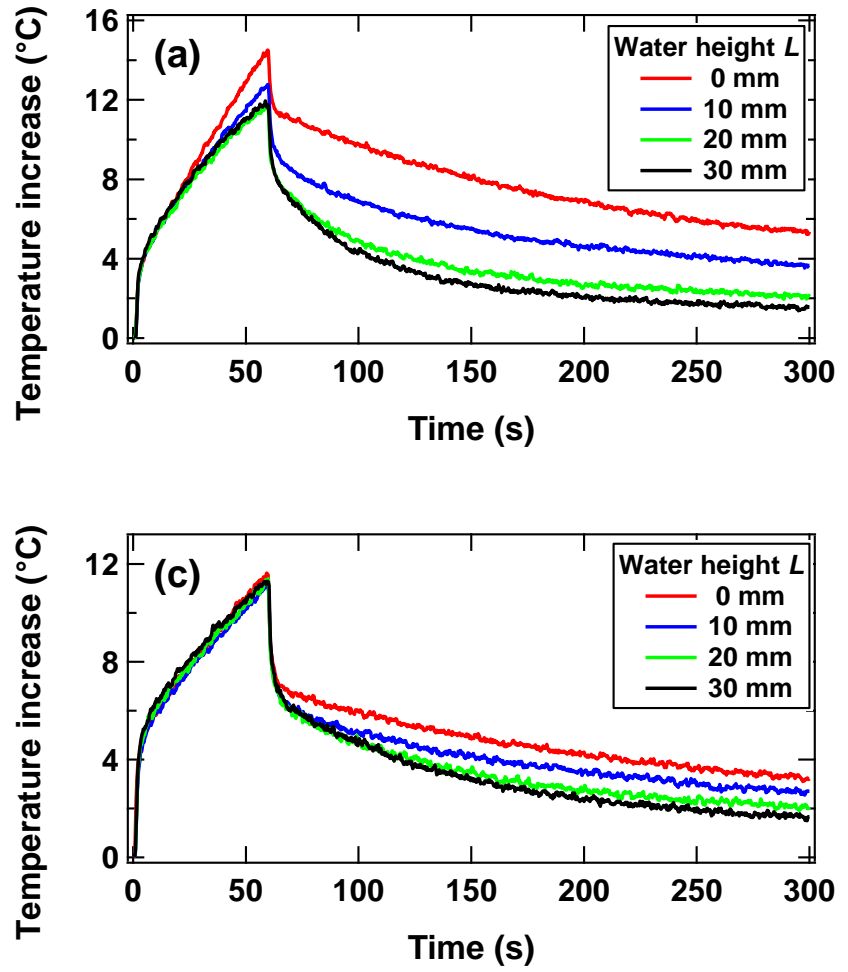
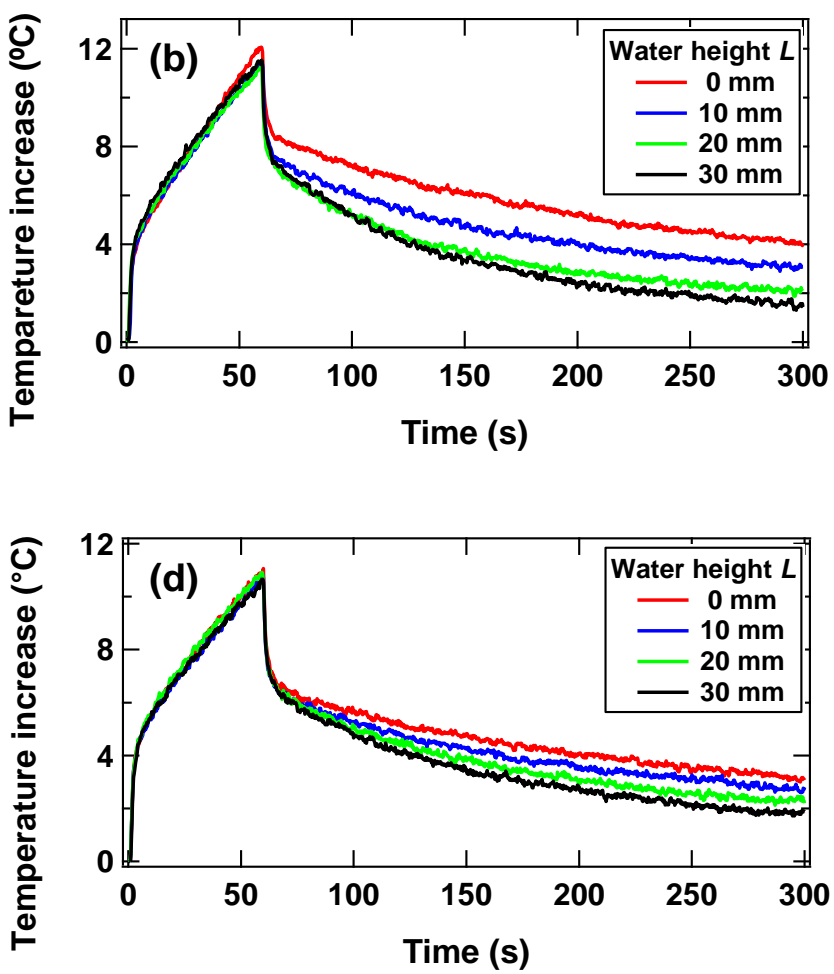

Fig. 4. Experimentally observed temperature increase as a function of time for each $L$ for (a) 100A-sch40, (b) sch80, (c) sch120, and (d) sch160.

\section{Estimation of remaining water}

\subsection{Data processing for the estimation}

To quantitatively estimate the values of $L$, we suggest a data processing method that was applied to the obtained temperature-time data. Figure 5 shows the procedure of the data processing. First, the temperature-time data after $65 \mathrm{~s}$ (i.e., 5 seconds after heating is stopped) is extracted (figure 5(b)). Next, the extracted data is displayed as a log-log graph (figure 5(c)). Then, the log-log data is approximated using a cubic polynomial, and the approximation curve is redisplayed as a linear graph (figure $5(\mathrm{~d})$ and (e)). Finally, the approximated data is normalized with the value at $65 \mathrm{~s}$ (figure $5(\mathrm{f})$ ). The normalization process is applied to eliminate the dependency of the amount of heat input. Figure 6 shows the experimental results after applying the processing along with the numerical results. The temperature change caused by the variation of $L$ is clearly observed also in the processed data. In the practical estimation, experimentally obtained temperature data is processed, and the $L$ value is estimated by compared the processed data with the numerical data as reference.

\subsection{Verification of the estimation procedure}

The water height $L$ was estimated from the experimentally obtained temperature-time data using the proposed data processing method. The estimation was performed in two ways: one is to compare the processed value at $150 \mathrm{~s}$, and the other is to compare the inclination between the values at 65 and $75 \mathrm{~s}$. Figure 7 shows the comparison of numerical and experimental results. By using these relations, the $L$ values were estimated as presented in Table 3 . The $L$ values were estimated with an error of $\pm 5 \mathrm{~mm}$ by both methods (using the values at 150 seconds showed a slightly higher accuracy). These results suggest that the proposed method is effective to roughly estimate the height of remaining water. 
(a)

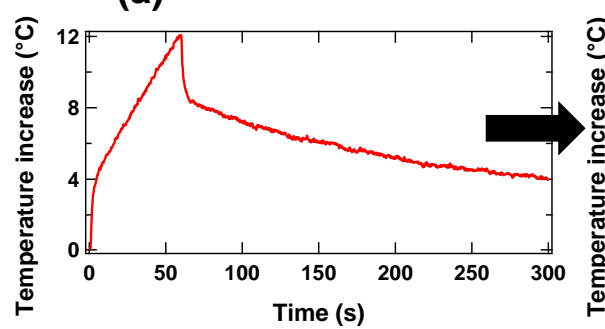

(d)

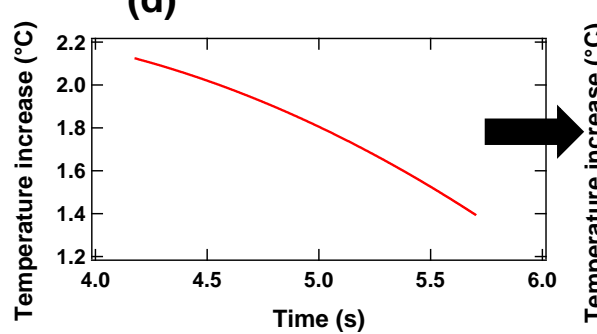

(b)

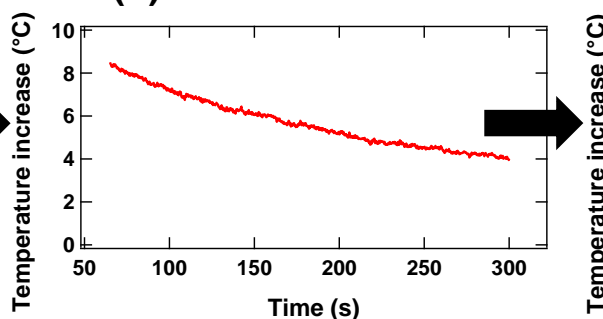

(e)

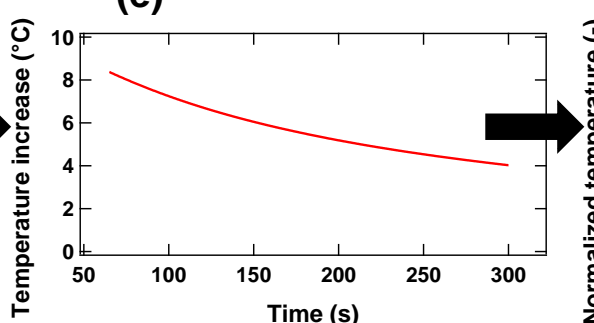

(c)

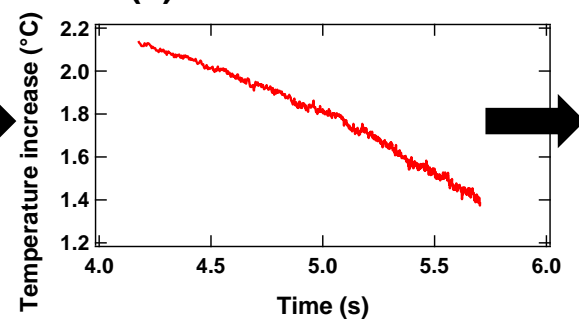

(f)

Fig. 5. Data processing procedure: (a) Original temperature-time data, (b) Extracting temperature-time data after $65 \mathrm{~s}$, (c) Transformation to a log-log graph, (d) Approximation with cubic polynomial, (e) Redisplayed as a linear graph, (f) Normalization by the value at $65 \mathrm{~s}$.
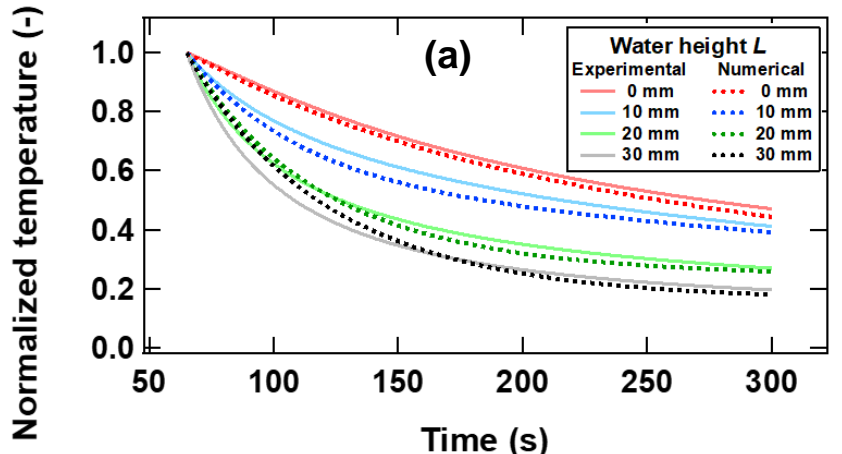

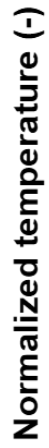

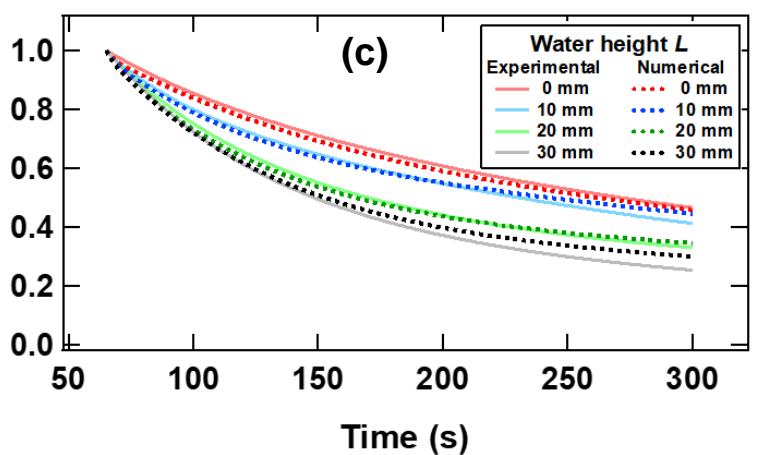

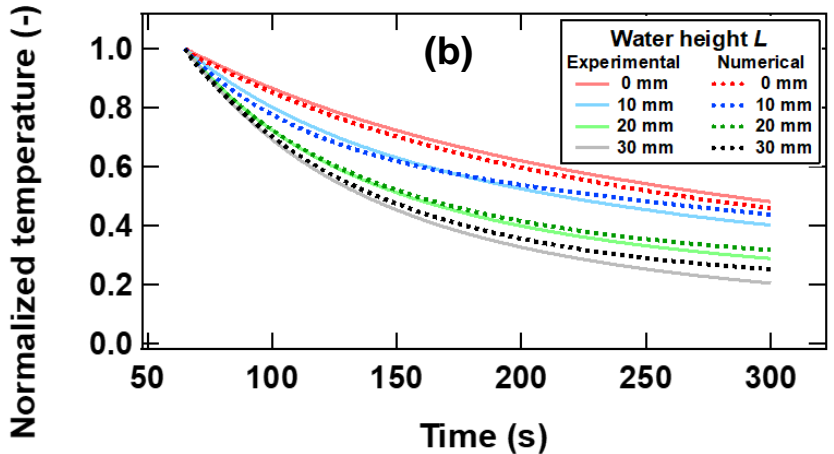

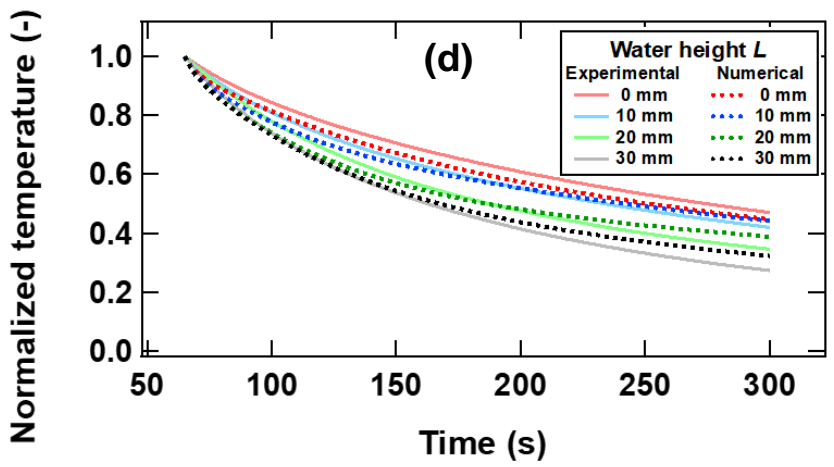

Fig. 6. Data processed experimental and numerical results for (a) 100A-sch40, (b) sch80, (c) sch120, and (d) sch160. 

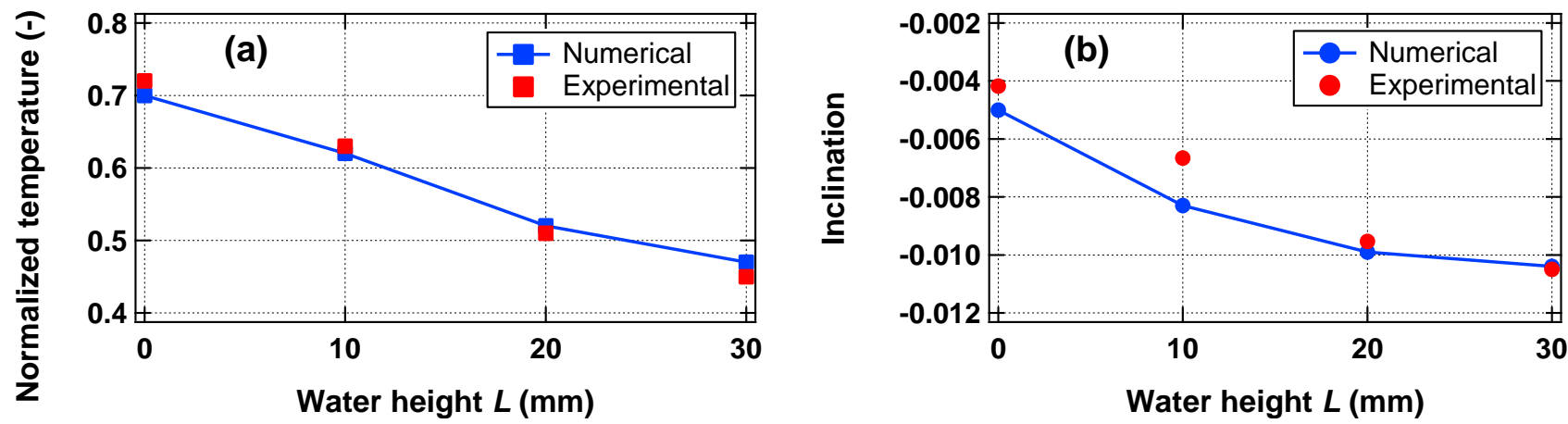

Fig. 7. Comparison between the numerical and experimental results (100A-sch80) after applying data processing, (a) comparison of value at $150 \mathrm{~s}$, and (b) comparison of Inclination between the values at 65 and 75 seconds.

Table 3. Estimated water height $L$ estimated by the two methods presented in figure 7.

\begin{tabular}{|c|cc|}
\hline Actual water height $L$ & (a) & (b) \\
\hline $0 \mathrm{~mm}$ & $-3 \mathrm{~mm}$ & $-3 \mathrm{~mm}$ \\
$10 \mathrm{~mm}$ & $8 \mathrm{~mm}$ & $5 \mathrm{~mm}$ \\
$20 \mathrm{~mm}$ & $22 \mathrm{~mm}$ & $17 \mathrm{~mm}$ \\
$30 \mathrm{~mm}$ & $33 \mathrm{~mm}$ & $32 \mathrm{~mm}$ \\
\hline
\end{tabular}

\section{Conclusions}

The effectiveness of active thermography to estimate of the remaining water quantity in the pipes was examined with a suggestion of a data processing procedure to determine the remaining water height. The numerical and experimental results showed that the observed surface temperature depends on the water height in the pipe, and the water height could be estimated by comparing the observed temperature after applying the data processing with the numerical reference data. These results suggest that the active thermography method could be an effective alternative way to inspect the remaining water in pipes without contact and remotely.

\section{REFERENCES}

[1] Oblak T.A., Dudek D.F., Smith J.R., "Ultrasonic liquid level measurement system”. US Patent no. 5,319,972, 1994.

[2] Lih, S.S., Bar-Cohen, Y., Lee, H.J., Takano, N., Bao, X. "Advanced signal processing for high temperatures health monitoring of condensed water height in steam pipes", Health Monitoring of Structural and Biological Systems 2013. Vol. 8695. International Society for Optics and Photonics, 2013.

[3] K. Ogawa., Japanese Patent Application publication no. 2012-251836, 2012.

[4] M. Suzuki., Japanese Patent Application publication no. 2018-025390, 2018. 